\title{
Identification of persistent and resolving subphenotypes of acute hypoxemic respiratory failure in two independent cohorts
}

\author{
Neha A. Sathe ${ }^{1 *}$ (D), Leila R. Zelnick ${ }^{2}$, Carmen Mikacenic ${ }^{1,3}$, Eric D. Morrell ${ }^{1}$, Pavan K. Bhatraju 1,4, \\ J. Brennan McNeil ${ }^{5}$, Susanna Kosamo ${ }^{6}$, Catherine L. Hough ${ }^{7}$, W. Conrad Liles ${ }^{4,8}$, Lorraine B. Ware ${ }^{5,9}$ and \\ Mark M. Wurfel ${ }^{1,4}$
}

\begin{abstract}
Background: Acute hypoxemic respiratory failure (HRF) is associated with high morbidity and mortality, but its heterogeneity challenges the identification of effective therapies. Defining subphenotypes with distinct prognoses or biologic features can improve therapeutic trials, but prior work has focused on ARDS, which excludes many acute HRF patients. We aimed to characterize persistent and resolving subphenotypes in the broader HRF population.

Methods: In this secondary analysis of 2 independent prospective ICU cohorts, we included adults with acute HRF, defined by invasive mechanical ventilation and $\mathrm{PaO}_{2}$-to- $\mathrm{FIO}_{2}$ ratio $\leq 300$ on cohort enrollment $(n=768$ in the discovery cohort and $n=1715$ in the validation cohort). We classified patients as persistent HRF if still requiring mechanical ventilation with $\mathrm{PaO}_{2}$-to- $\mathrm{FIO}_{2}$ ratio $\leq 300$ on day 3 following ICU admission, or resolving HRF if otherwise. We estimated relative risk of 28-day hospital mortality associated with persistent HRF, compared to resolving HRF, using generalized linear models. We also estimated fold difference in circulating biomarkers of inflammation and endothelial activation on cohort enrollment among persistent HRF compared to resolving HRF. Finally, we stratified our analyses by ARDS to understand whether this was driving differences between persistent and resolving HRF.

Results: Over $50 \%$ developed persistent HRF in both the discovery $(n=386)$ and validation $(n=1032)$ cohorts. Persistent HRF was associated with higher risk of death relative to resolving HRF in both the discovery $(1.68$-fold, $95 \% \mathrm{Cl}$ $1.11,2.54)$ and validation cohorts (1.93-fold, $95 \% \mathrm{Cl} 1.50,2.47)$, after adjustment for age, sex, chronic respiratory illness, and acute illness severity on enrollment (APACHE-III in discovery, APACHE-II in validation). Patients with persistent HRF displayed higher biomarkers of inflammation (interleukin-6, interleukin-8) and endothelial dysfunction (angiopoietin-2) than resolving HRF after adjustment. Only half of persistent HRF patients had ARDS, yet exhibited higher mortality and biomarkers than resolving HRF regardless of whether they qualified for ARDS.

Conclusion: Patients with persistent HRF are common and have higher mortality and elevated circulating markers of lung injury compared to resolving HRF, and yet only a subset are captured by ARDS definitions. Persistent HRF may represent a clinically important, inclusive target for future therapeutic trials in HRF.
\end{abstract}

Keywords: Acute hypoxemic respiratory failure, Mechanical ventilation, Acute lung injury, ARDS, Endophenotypes

\footnotetext{
*Correspondence: nas212@uw.edu

${ }^{1}$ Division of Pulmonary, Critical Care and Sleep Medicine, Department of Medicine, University of Washington, 325 9th Avenue, Box \# 359640, Seattle, WA 98104, USA

Full list of author information is available at the end of the article
}

\section{Background}

Acute hypoxemic respiratory failure (HRF) is associated with extended hospital length of stay, functional disability, and increased mortality, but care remains supportive 
[1-5]. One challenge in identifying effective therapeutics for a broadly defined syndrome like acute HRF is heterogeneity in treatment response and prognosis [6]. Defining reliable subsets of patients with high likelihood of disease-related events or differential treatment responses (often termed subphenotypes) can help target clinical care and trial enrollment to patients most likely to benefit $[7,8]$. Previous efforts to identify subphenotypes of respiratory failure have largely centered on acute respiratory distress syndrome (ARDS), which represents less than a quarter of patients on mechanical ventilation, and two-thirds of patients with acute HRF [9-12]. Reliably diagnosing ARDS is also challenging, largely driven by variability in the interpretation and sensitivity of the chest radiograph [13-17]. As such, characterizing subphenotypes of acute HRF is a newly identified research priority by the National Heart, Lung, and Blood Institute with potential to expand inclusion criteria for trials [818].

One area of heterogeneity in HRF is the early clinical trajectory. The majority of patients with respiratory failure requiring mechanical ventilation are weaned off within three days [19], while others may develop worsening lung injury and/or excessive fibroproliferation contributing to prolonged need for mechanical ventilation and hypoxemia [20]. Using trajectories of clinical and biologic data to classify subphenotypes has helped identify patients at high risk for poor outcomes in such heterogeneous conditions as chronic obstructive pulmonary disease, pneumonia, and acute kidney injury, and can help determine early biologic events that contribute to subsequent clinical course [10, 21-26].

Our overall goal was to define and characterize a novel subphenotype of acute HRF using persistence of respiratory failure at day 3 following the initiation of mechanical ventilation (persistent HRF). The primary objective of this study was to estimate mortality of patients with persistent HRF compared to patients whose respiratory failure and need for mechanical ventilation resolves (resolving HRF). The secondary objective was to compare circulating biomarkers previously linked to severity and outcomes in lung injury and profile the early biologic differences between patients with persistent and resolving HRF. In exploratory analyses, we examined relationships between persistent/resolving HRF and ARDS. In particular, we were interested in characterizing the clinical and biologic features of patients with acute HRF who do not fulfill ARDS criteria, a population that is relatively understudied [27].

\section{Methods}

\section{Discovery and validation cohorts}

We performed a secondary analysis of two prospective observational cohort studies of adults admitted to the intensive care unit (ICU). The discovery cohort was enrolled from medical and surgical ICUs at Harborview Medical Center (Seattle, WA) between 2006 and 2010. Patients were enrolled within 24 hours of ICU admission if meeting criteria for the systemic inflammatory response syndrome [28-30]. Exclusion criteria included admission for trauma; admission for intracranial hemorrhage; severe immunosuppression; and active cancer diagnosis. The validation cohort was enrolled from the medical, surgical, or trauma ICUs at Vanderbilt University Medical Center (Nashville, TN) on the day following admission, from 2006 to 2020, as part of the Validating Acute Lung Injury Biomarkers for Diagnosis (VALID) study [31]. Exclusion criteria included severe chronic lung disease on home supplemental oxygen; cardiac arrest prior to admission; and anticipated discharge from ICU on the day of enrollment. The studies were approved by IRBs at University of Washington and Vanderbilt University. (Additional file 1 has additional cohort description.)

\section{Study definitions}

We restricted analyses to patients with acute HRF, defined as new invasive mechanical ventilation and $\mathrm{PaO}_{2}$-to- $\mathrm{FIO}_{2}$ ratio $\left(\mathrm{PaO}_{2}: \mathrm{FIO}_{2}\right) \leq 300$ at study enrollment. If $\mathrm{PaO}_{2}: \mathrm{FIO}_{2}$ was unavailable, we used $\mathrm{SpO}_{2}$-to- $\mathrm{FIO}_{2}$ ratio $\leq 315$ [32]. (Additional file 1 has additional details.)

Patients with acute HRF were further classified as persistent or resolving. Persistent HRF was defined by ongoing need for invasive mechanical ventilation and $\mathrm{PaO}_{2}: \mathrm{FIO}_{2} \leq 300$ on day 3 following ICU admission. Patients with transient improvements before day 3 (e.g., patients extubated but reintubated, or $\mathrm{PaO}_{2}: \mathrm{FIO}_{2}>300$ on day 2) were still classified as persistent HRF as long as they met criteria on day 3 . Patients who were extubated or who had $\mathrm{PaO}_{2}: \mathrm{FIO}_{2}>300$ on day 3 were classified as resolving HRF. All $\mathrm{PaO}_{2}$ measurements were obtained as part of clinical care, and we used the lowest value of $\mathrm{PaO}_{2}: \mathrm{FIO}_{2}$ for each ICU day. To understand how classifying persistent $\mathrm{HRF}$ at different early time points altered our results, we performed sensitivity analyses redefining persistent HRF on day 2 after admission (which may fall within the eligibility period of prior trials in respiratory failure) and day 4 (which we hypothesized may better delineate differences in underlying biology) [33-35]. Patients who died before qualifying for persistent HRF were excluded in primary analyses but included in sensitivity analyses. They were classified as persistent HRF if they met the specified criteria on the day of death.

ARDS was defined by the Berlin criteria [36]. Each chest radiograph was reviewed by two Critical Care physicians who came to a consensus on the presence of bilateral opacities consistent with ARDS. The primary 
outcome was cumulative hospital mortality 28 days following enrollment.

\section{Biomarker measurements}

In both cohorts, plasma samples were collected at enrollment for measurement of biomarkers associated with the development of acute lung injury and ARDS [28, 31]. In the discovery cohort, we measured markers of inflammation and apoptosis (interleukin-6 [IL-6], interleukin-8 [IL-8], soluble tumor necrosis factor receptor-1 [sTNFR-1], soluble Fas [sFas], interleukin-17A [IL-17A], granulocyte colony-stimulating factor [G-CSF] ) as well as markers of endothelial dysfunction/activation (angiopoietin-2 [Ang-2], angiopoietin-1 [Ang-1], and soluble vascular cell adhesion protein-1 [sVCAM-1]) using a electrochemiluminiscent immunoassay (MesoScale Discovery, Rockville, MD). Of these markers, IL-6, IL-8, sTNFR-1, and Ang-2 were also measured in the validation cohort. IL- 6 and IL- 8 were measured on the same platform, while sTNFR-1 and Ang- 2 were measured by ELISA (R\&D Systems, Minneapolis, MN).

\section{Statistical analyses}

We reported demographics, baseline comorbidities, ICU conditions, and outcomes by persistent and resolving HRF. Continuous variables were reported as medians with interquartile ranges and compared using Mann-Whitney U tests, while categorical variables were reported as counts with percentage and compared using Chi-squared tests.

Our primary analysis estimated relative risk (RR) of mortality associated with persistent HRF compared to resolving HRF using generalized linear models with Poisson distribution and robust standard errors [37]. In adjusted models, we included age, sex, chronic respiratory disease, and either baseline $\mathrm{PaO}_{2}: \mathrm{FIO}_{2}$ or modified acute physiology and chronic health evaluation (APACHE) score as prespecified confounders. We used APACHE-III in the discovery cohort and APACHE-II in the validation cohort $[38,39]$. We did not include both $\mathrm{PaO}_{2}: \mathrm{FIO}_{2}$ and APACHE score in the same model to avoid multicollinearity. To further select for a population of comparable HRF severity at baseline, we performed analyses restricting to patients with $\mathrm{PaO}_{2}: \mathrm{FIO}_{2}<150$ and another excluding patients with chronic lung disease.

In our secondary analysis, we used linear regression with adjustments as above to estimate the fold difference in geometric mean concentrations of each biomarker between persistent and resolving HRF. We used geometric means and $\log _{2}$-transformed biomarker measurements due to right skew.

We performed two exploratory analyses. First, we examined whether ARDS, identified at any point by ICU day 3, was driving differences between persistent and resolving HRF. We stratified persistent/resolving HRF by ARDS ( \pm ARDS) to create 4 groups: (1) resolving HRF/ARDS, (2) resolving HRF/+ARDS, (3) persistent HRF/ARDS, and (4) persistent HRF/+ARDS. We compared $R R$ of mortality in each group to the reference of resolving HRF/-ARDS using the methods from our primary analysis. In a second exploratory analysis, we examined whether previously described hyperinflammatory and hypoinflammatory subphenotypes have any relationship to persistent HRF [9, 40-43]. These subphenotypes have been independently validated in ARDS and HRF, and were associated with distinct prognoses. We classified patients as hypoinflammatory or hyperinflammatory using a logistic regression model of plasma sTNFR-1, IL-8, and bicarbonate as previously reported [44], and then compared the proportion of patients who developed persistent HRF in each group.

Observations with missing covariates were excluded from each model. Analyses were conducted in STATA (version 16.0).

\section{Results}

\section{Cohort description}

We identified 768 patients with acute HRF in the discovery cohort, and 1715 patients in the validation cohort (Additional file 1: Figures S1-S2). In the discovery cohort, 386 patients (50\%) developed persistent HRF; in the validation cohort, 1032 (60\%) patients developed persistent HRF. Baseline characteristics and outcomes by persistent and resolving HRF are summarized in Table 1. Patients with persistent HRF had higher illness severity and a higher proportion of ARDS on enrollment compared to patients with resolving HRF. Patients with persistent HRF also had fewer VFD and longer hospital lengths of stay, with the median differences exceeding what was expected based on how persistent HRF was defined. We had limited data regarding initial ventilator management (Additional file 1: Table S1), but noted patients with persistent HRF were treated with modestly higher $\mathrm{FIO}_{2}$ than patients with resolving HRF in the discovery cohort, and all patients in both cohorts were on at least $5 \mathrm{~cm} \mathrm{H}_{2} \mathrm{O}$ positive end-expiratory pressure.

\section{Mortality in persistent/resolving HRF}

Mortality was significantly higher among patients with persistent HRF, compared to those with resolving HRF ( $20 \%$ vs. $8 \%$ in discovery; $22 \%$ vs. $10 \%$ in validation). Adjusting for age, sex, chronic respiratory disease, and APACHE-III, persistent HRF carried a 1.68-fold higher $(95 \%$ CI 1.11, 2.54) risk of death in the discovery cohort (Table 2). In the validation cohort, persistent HRF was associated with a 1.93 -fold higher (95\% CI 1.50, 2.47) 
Table 1 Cohort descriptions by persistent and resolving hypoxemic respiratory failure

\begin{tabular}{|c|c|c|c|c|c|c|}
\hline & \multicolumn{3}{|l|}{ Discovery cohort } & \multicolumn{3}{|l|}{ Validation cohort } \\
\hline & Resolving $N=382$ & Persistent $N=386$ & $P$ value & Resolving $N=683$ & Persistent $N=1032$ & $P$ value \\
\hline \multicolumn{7}{|l|}{ Demographics } \\
\hline Age, years & $54(45-63)$ & $53(44-64)$ & 0.780 & $55(41-66)$ & $53(41-64)$ & 0.131 \\
\hline Female sex & $144(38 \%)$ & $129(33 \%)$ & 0.216 & $264(39 \%)$ & $357(35 \%)$ & 0.087 \\
\hline Race & & & 0.013 & & & 0.004 \\
\hline White & $271(76 \%)$ & $291(80 \%)$ & & $573(84 \%)$ & $910(88 \%)$ & \\
\hline Black/African American & $50(14 \%)$ & $26(7 \%)$ & & $99(14 \%)$ & $98(10 \%)$ & \\
\hline Other & $61(16 \%)$ & $69(18 \%)$ & & $11(2 \%)$ & $24(2 \%)$ & \\
\hline \multicolumn{7}{|l|}{ Baseline comorbidities } \\
\hline Diabetes & $113(30 \%)$ & $112(29 \%)$ & 0.863 & $186(27 \%)$ & $258(25 \%)$ & 0.301 \\
\hline Cirrhosis & $44(12 \%)$ & $44(11 \%)$ & 0.959 & $61(9 \%)$ & $74(7 \%)$ & 0.185 \\
\hline Chronic respiratory disease & $60(16 \%)$ & $88(23 \%)$ & 0.013 & $134(20 \%)$ & $122(12 \%)$ & $<0.001$ \\
\hline Heart Failure & $41(11 \%)$ & $43(11 \%)$ & 0.857 & $74(11 \%)$ & $83(8 \%)$ & 0.050 \\
\hline Alcohol Use Disorder & $115(30 \%)$ & $135(35 \%)$ & 0.150 & 139 (20\%) & $240(23 \%)$ & 0.156 \\
\hline \multicolumn{7}{|l|}{ ICU events on enrollment } \\
\hline Type of ICU & & & 0.423 & & & $<0.001$ \\
\hline Medical & $260(68 \%)$ & $273(71 \%)$ & & $305(45 \%)$ & $409(40 \%)$ & \\
\hline Surgical & $122(32 \%)$ & $113(29 \%)$ & & 164 (24\%) & $192(19 \%)$ & \\
\hline Trauma & $0(0 \%)$ & $0(0 \%)$ & & $210(31 \%)$ & $429(42 \%)$ & \\
\hline Shock & $87(23 \%)$ & 191 (49\%) & $<0.001$ & $261(38 \%)$ & $532(52 \%)$ & $<0.001$ \\
\hline Sepsis & $298(78 \%)$ & $330(85 \%)$ & 0.007 & $261(38 \%)$ & $475(46 \%)$ & 0.001 \\
\hline Pneumonia & $81(21 \%)$ & $143(37 \%)$ & $<0.001$ & $198(29 \%)$ & $385(37 \%)$ & $<0.001$ \\
\hline $\begin{array}{l}\text { Acute respiratory distress syndrome } \\
\text { Illness severity on enrollment }\end{array}$ & $51(13 \%)$ & $124(32 \%)$ & $<0.001$ & $145(21 \%)$ & $393(38 \%)$ & $<0.001$ \\
\hline $\mathrm{PaO}_{2}$-to- $\mathrm{FIO}_{2}$ ratio & $190(138-254)$ & $128(85-207)$ & $<0.001$ & $187(136-236)$ & $147(97-205)$ & $<0.001$ \\
\hline SOFA & $4(3-6)$ & $5(4-7)$ & $<0.001$ & $8(7-10)$ & $10(8-11)$ & $<0.001$ \\
\hline APACHE-III & $53(36-72)$ & $69(49-90)$ & $<0.001$ & n.a & n.a & \\
\hline APACHE-II & n.a & n.a & & $26(21-31)$ & $29(23-34)$ & $<0.001$ \\
\hline \multicolumn{7}{|l|}{ Outcomes } \\
\hline Ventilator free days & $25(23-26)$ & $15(0-21)$ & $<0.001$ & $25(23-26)$ & $17(0-22)$ & $<0.001$ \\
\hline Hospital length of stay, days & $10(6-19)$ & $19(12-32)$ & $<0.001$ & $10(7-18)$ & $16(10-26)$ & $<0.001$ \\
\hline Mortality & $31(8 \%)$ & 77 (20\%) & $<0.001$ & $70(10 \%)$ & $230(22 \%)$ & $<0.001$ \\
\hline
\end{tabular}

ICU intensive care unit, APACHE acute physiology and chronic health evaluation, SOFA sequential organ failure assessment, n.a. not applicable. Continuous variables are expressed as median (interquartile range) and categorical variables are expressed as number (percentage). $P$ values correspond to Mann-Whitney $U$ tests for continuous variables and Chi-squared tests for categorical variables

risk of death in a similarly adjusted model. Fewer than $5 \%$ of patients died before day 3 in either cohort and were excluded from primary analyses; the findings were similar when we included these patients (Additional file 1: Table S2). The association between persistent HRF and mortality was robust to other sensitivity analyses, including redefining persistent HRF at day 2 and day 4 , as well as restricting the population to enrollment $\mathrm{PaO}_{2}: \mathrm{FIO}_{2}<150$ and those without chronic lung disease (Additional file 1: Tables S3-S6).

\section{Circulating biomarkers in persistent/resolving HRF}

We next compared enrollment biomarker profiles to see whether persistent and resolving HRF exhibited distinctive early patterns of inflammation and/or lung injury (Additional file 1: Tables S7-S8). In the discovery cohort, biomarkers of inflammation were higher in patients with persistent HRF compared to resolving HRF, including IL-6 (2.44-fold [95\% CI 1.84, 3.23]), G-CSF (1.85fold [95\% CI 1.43, 2.40]), IL-8 (1.64-fold [95\% CI 1.30, 2.07]), and sTNFR-1 (1.25-fold [95\% CI 1.08, 1.46]) even 
Table 2 Relative risk of mortality associated with persistent hypoxemic respiratory failure

\begin{tabular}{|c|c|c|c|c|}
\hline & \multirow{2}{*}{$\begin{array}{l}\text { Deaths } \\
N(\%)\end{array}$} & \multicolumn{3}{|c|}{ Relative risk (95\% confidence interval) } \\
\hline & & Unadjusted & Model A & Model B \\
\hline \multicolumn{5}{|c|}{ Discovery cohort } \\
\hline Resolving & $31(8 \%)$ & $\begin{array}{l}1.00 \text { (refer- } \\
\text { ence) }\end{array}$ & $\begin{array}{l}1.00 \text { (refer- } \\
\text { ence) }\end{array}$ & 1.00 (reference) \\
\hline Persistent & $77(20 \%)$ & $\begin{array}{l}2.46(1.66 \\
3.64)^{\mathrm{a}}\end{array}$ & $\begin{array}{l}2.34(1.56 \\
3.49)^{\mathrm{a}}\end{array}$ & $1.68(1.11,2.54)^{\mathrm{b}}$ \\
\hline \multicolumn{5}{|c|}{ Validation cohort } \\
\hline Resolving & $70(10 \%)$ & $\begin{array}{l}1.00 \text { (refer- } \\
\text { ence) }\end{array}$ & $\begin{array}{l}1.00 \text { (refer- } \\
\text { ence) }\end{array}$ & 1.00 (reference) \\
\hline Persistent & $230(22 \%)$ & $\begin{array}{l}2.17(1.69 \\
2.79)^{\mathrm{a}}\end{array}$ & $\begin{array}{l}2.08(1.58 \\
2.76)^{\mathrm{a}}\end{array}$ & $1.93(1.50,2.47)^{\mathrm{a}}$ \\
\hline
\end{tabular}

Mortality is in-hospital mortality 28 days after enrollment

Model A: adjusted for age, sex, chronic respiratory disease, and $\mathrm{PaO}_{2}$-to- $\mathrm{FIO}_{2}$ ratio on enrollment

Model B: adjusted for age, sex, chronic respiratory disease, and modified acute physiology and chronic health evaluation on enrollment (APACHE-III score in discovery cohort and APACHE-II score in validation cohort)

${ }^{\mathrm{a}} p<0.001{ }^{\mathrm{b}} p<0.05$

after adjusting for age, sex, chronic respiratory disease, and APACHE (Fig. 1). A marker of apoptotic pathways, sFas, was 1.11 -fold higher ( $95 \%$ CI 1.02, 1.20) in persistent HRF compared to resolving HRF. Ang-2, reflecting endothelial injury, was 1.49-fold higher (95\% CI 1.27, 1.74) in persistent HRF.

We replicated the finding that persistent HRF is associated with greater inflammation (higher IL-6, IL-8) and endothelial injury (higher Ang-2) compared to resolving HRF in the validation cohort (Fig. 1). Only sTNFR-1 was found to be $14 \%$ lower among persistent HRF compared to resolving HRF (0.86-fold, 95\% CI 0.76, 0.97) in APACHE-adjusted models; however, the difference was not significant in other unadjusted or adjusted analyses (Additional file 1: Tables S7-S8).

Sensitivity analyses produced similar results (Additional file 1: Tables S9-S13). Of note, redefining persistent HRF at day 2 attenuated differences in some biomarkers, while redefining persistent HRF at day 4 and restricting analyses to patients with $\mathrm{PaO}_{2}: \mathrm{FIO}_{2}<150$ magnified biologic differences between groups.

\section{Persistent/resolving HRF stratified by ARDS}

Additional file 1: Table S14 lists features of patients with persistent and resolving HRF, stratified by whether or not they met ARDS criteria at any point by day 3. Of note, half of patients with persistent HRF did not meet ARDS criteria.

In the discovery cohort, mortality was highest among persistent $\mathrm{HRF} /+\mathrm{ARDS}$ (25\%), followed by persistent HRF/-ARDS (15\%), resolving HRF/+ARDS (10\%), and resolving HRF/-ARDS (7\%) (Table 3). Persistent HRF both with and without ARDS were associated with a higher risk for death compared to resolving HRF/ARDS, although adjustment for APACHE-III attenuated estimates. Resolving HRF/+ARDS did not have a significantly higher risk for death compared to resolving HRF/-ARDS. In the validation cohort, all 3 comparator

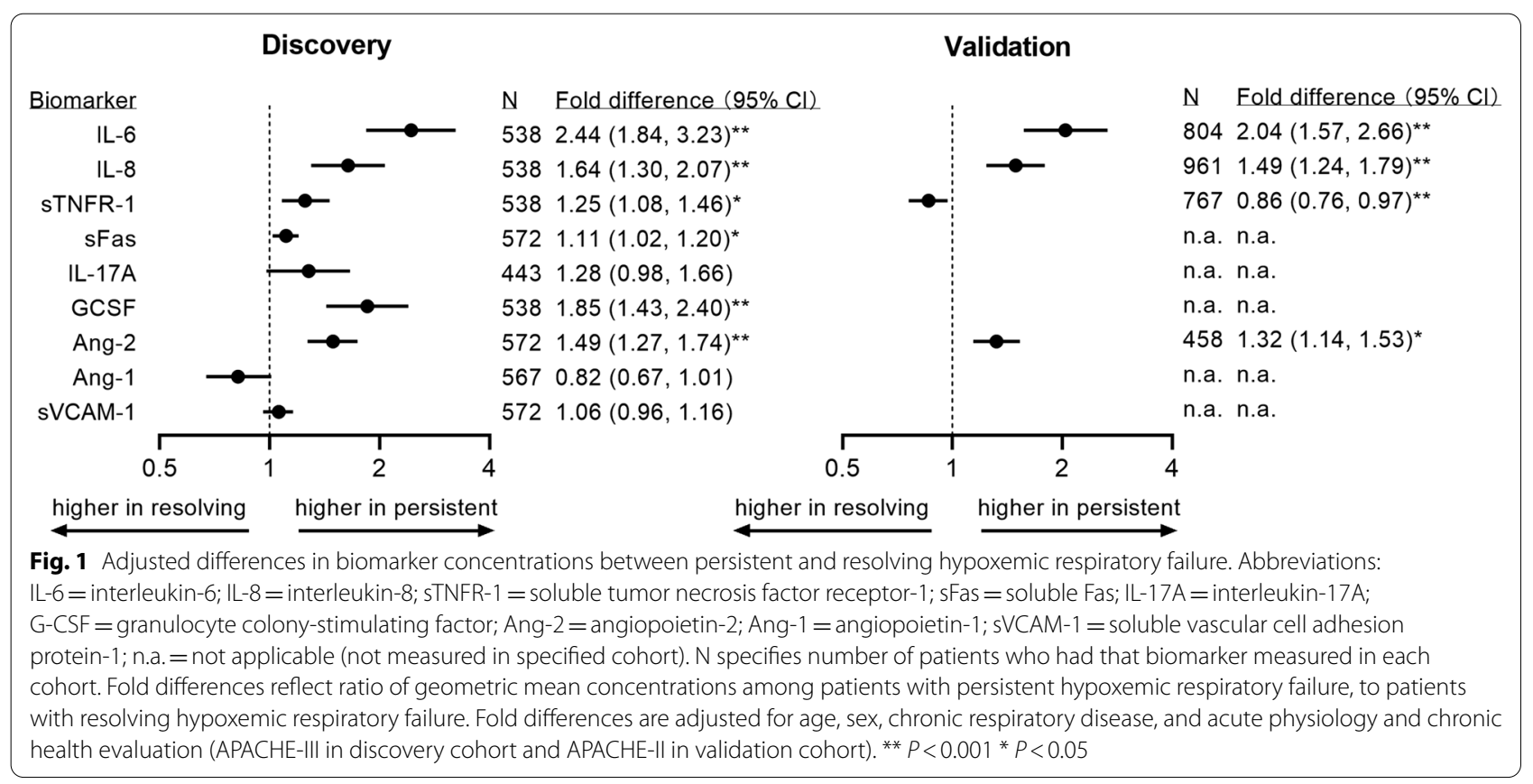


Table 3 Relative risk of mortality associated with persistent and resolving hypoxemic respiratory failure, stratified by acute respiratory distress syndrome ( \pm ARDS)

\begin{tabular}{|c|c|c|c|c|c|c|}
\hline & & \multirow{2}{*}{$\begin{array}{l}\text { Total } \\
N\end{array}$} & \multirow{2}{*}{$\begin{array}{l}\text { Deaths } \\
N(\%)\end{array}$} & \multicolumn{3}{|c|}{ Relative risk (95\% confidence interval) } \\
\hline & & & & Unadjusted & Model A & Model B \\
\hline \multicolumn{7}{|c|}{ Discovery cohort } \\
\hline \multirow[t]{2}{*}{ Resolving } & - ARDS & 296 & $22(7 \%)$ & 1.00 (reference) & 1.00 (reference) & 1.00 (reference) \\
\hline & + ARDS & 86 & $9(10 \%)$ & $1.41(0.67,2.94)$ & $1.36(0.65,2.83)$ & $0.96(0.49,1.89)$ \\
\hline \multirow[t]{2}{*}{ Persistent } & - ARDS & 186 & $28(15 \%)$ & $2.03(1.19,3.43)^{b}$ & $2.03(1.20,3.43)^{b}$ & $1.48(0.86,2.52)$ \\
\hline & + ARDS & 200 & $49(25 \%)$ & $3.30(2.06,5.28)^{\mathrm{a}}$ & $3.09(1.91,5.02)^{\mathrm{a}}$ & $1.81(1.07,3.08)^{c}$ \\
\hline \multicolumn{7}{|c|}{ Validation cohort } \\
\hline \multirow[t]{2}{*}{ Resolving } & - ARDS & 515 & $37(7 \%)$ & 1.00 (reference) & 1.00 (reference) & 1.00 (reference) \\
\hline & + ARDS & 168 & $33(20 \%)$ & $2.73(1.77,4.23)^{\mathrm{a}}$ & $2.48(1.53,4.02)^{\mathrm{a}}$ & $2.50(1.65,3.80)^{\mathrm{a}}$ \\
\hline \multirow[t]{2}{*}{ Persistent } & - ARDS & 559 & $114(20 \%)$ & $2.84(2.00,4.03)^{\mathrm{a}}$ & $2.59(1.77,3.79)^{\mathrm{a}}$ & $2.52(1.78,3.58)^{\mathrm{a}}$ \\
\hline & +sARDS & 473 & $116(25 \%)$ & $3.41(2.41,4.84)^{\mathrm{a}}$ & $3.19(2.17,4.69)^{\mathrm{a}}$ & $2.93(2.07,4.16)^{a}$ \\
\hline
\end{tabular}

Mortality is in-hospital mortality 28 days after enrollment

+ARDS refers to patients who were adjudicated as ARDS at any point by ICU day 3; -ARDS are patients who were not adjudicated to have ARDS by ICU day 3

Model A: adjusted for age, sex, chronic respiratory disease, and $\mathrm{PaO}_{2}$-to- $\mathrm{FIO}_{2}$ ratio on enrollment

Model B: adjusted for age, sex, chronic respiratory disease, and modified acute physiology and chronic health evaluation on enrollment (APACHE-III score in discovery cohort and APACHE-II score in validation cohort)

${ }^{\mathrm{a}} p<0.001{ }^{\mathrm{b}} p<0.01{ }^{\mathrm{c}} p<0.05$

groups had over twofold higher risk for death than resolving HRF/-ARDS.

Patients with persistent HRF, both with and without ARDS, exhibited similar biomarker profiles characterized by inflammation and endothelial injury (Additional file 1: Table S15). IL-6, IL-8, and Ang-2 concentrations were significantly higher among persistent HRF/+ARDS and persistent HRF/-ARDS in both discovery and validation cohorts, compared to resolving HRF/-ARDS (Fig. 2). In contrast, the biomarker profile among patients with resolving $\mathrm{HRF} /+\mathrm{ARDS}$ was not significantly different from resolving HRF/-ARDS. sTNFR-1 was lower among both +ARDS strata compared to resolving HRF/-ARDS in the validation cohort, but this difference was not seen in discovery.

Furthermore, we were interested in comparing resolving HRF/+ARDS to persistent HRF/-ARDS, expecting some elevations in lung injury biomarkers among +ARDS patients. We performed pairwise comparisons across all 4 strata with Bonferroni correction (Additional file 1: Table S16). We found persistent HRF/-ARDS patients consistently had higher markers of inflammation and endothelial dysfunction, while resolving HRF/+ARDS had higher Ang-1, a marker of endothelial stabilization.

\section{Persistent/resolving HRF and hyper/hypoinflammatory subphenotypes}

We classified the subset of patients with bicarbonate, IL-8, and sTNFR-1 measured as hypoinflammatory or hyperinflammatory (Additional file 1: Figure S3). The distribution and features of patients in each were consistent with prior descriptions of these subphenotypes in both ARDS and non-ARDS respiratory failure [9, 40-43], with mortality among hyperinflammatory patients higher than hypoinflammatory patients (Additional file 1: Table S17).

Patients with hyperinflammatory HRF were more likely to develop persistent HRF compared to patients with hypoinflammatory HRF in the discovery $(61 \%$ vs. $45 \%$, $P=0.001)$ and validation cohorts $(79 \%$ vs. $63 \% P<0.001)$. However, since few patients were classified as hyperinflammatory, far more hypoinflammatory patients than hyperinflammatory developed persistent HRF.

\section{Discussion}

In two independent cohorts of acute HRF, we found that patients with persistent HRF 3 days following ICU admission had a markedly higher risk for subsequent in-hospital mortality compared to patients with resolving HRF, even after adjustment for initial illness severity. Cumulative incidence of persistent HRF was over $50 \%$ in both cohorts, which included a large, diverse population of patients from medical, surgical, and trauma ICUs. Patients with persistent HRF had evidence of greater systemic inflammation and endothelial dysfunction/ activation on enrollment compared to resolving HRF. Finally, we saw that these findings were not driven only by patients with ARDS; rather, patients with persistent HRF who did not meet ARDS criteria represented a prevalent group with poor prognosis and elevated markers 


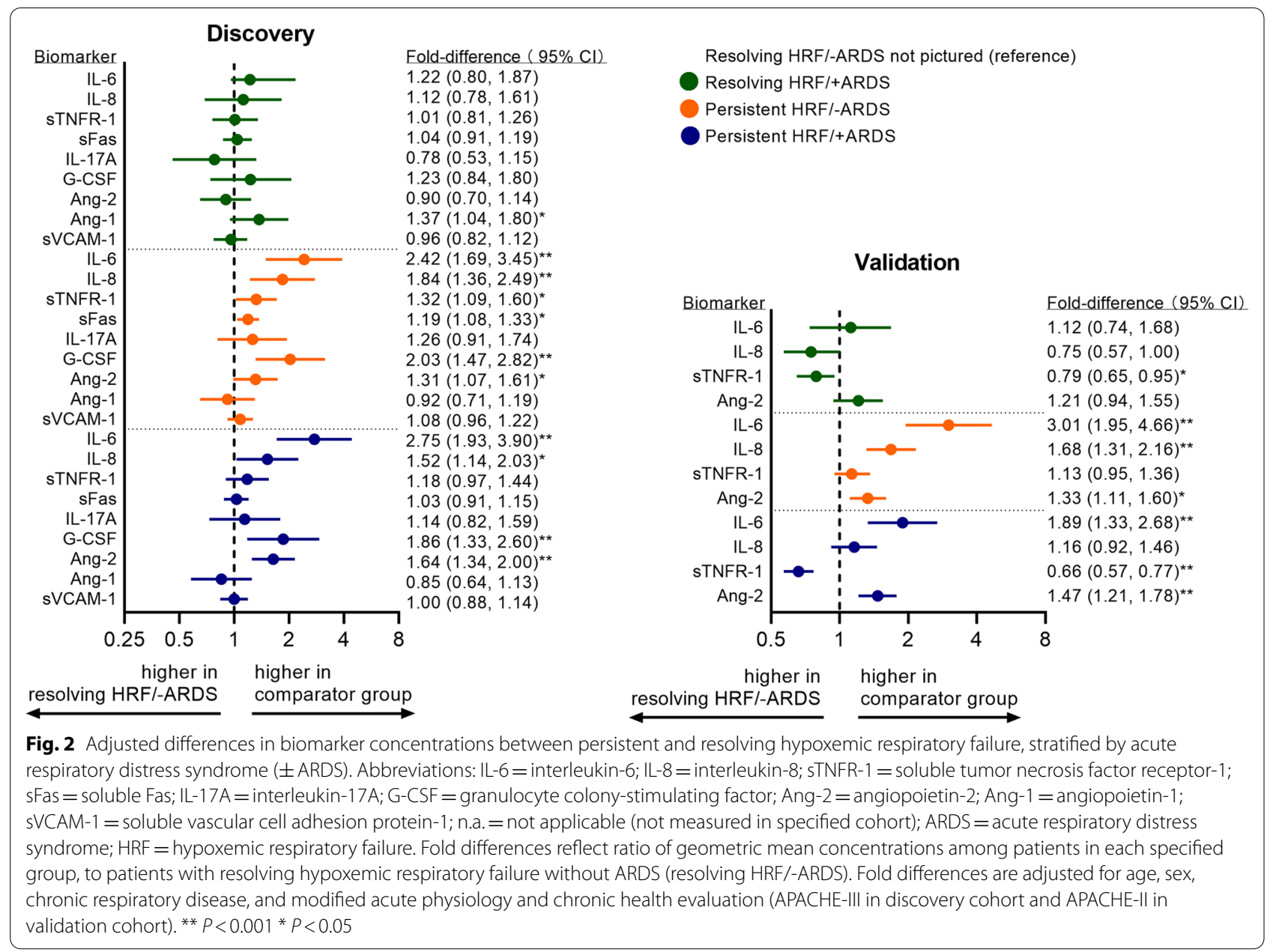

of lung injury. Overall, this work illustrates that ARDS is not merely one end of the spectrum of disease severity in acute HRF and that characterizing clinical and biologic features associated with persistent HRF regardless of ARDS diagnosis may generate new insight into what drives outcomes in respiratory failure.

We observed significant differences in mortality, VFD, and length of stay between persistent and resolving HRF. While the findings may in part reflect the milder illness among patients with resolving HRF at enrollment, we hypothesize patients with persistent and resolving HRF have other underlying reasons to explain differing trajectories. Supporting this hypothesis, the risk of death was different even after adjusting for baseline $\mathrm{PaO}_{2}: \mathrm{FIO}_{2}$ and APACHE score. Furthermore, our sensitivity analysis of patients with $\mathrm{PaO}_{2}: \mathrm{FIO}_{2}<150$ diminished differences in initial illness severity while strengthening the effect of persistent HRF on subsequent mortality. In fact, over half of the resolving HRF patients in our study had $\mathrm{PaO}_{2}: \mathrm{FIO}_{2}<150$ at baseline, indicating that many patients who begin their ICU course with serious respiratory failure improve quickly. The prognostic value of this early trajectory, and the disconnect between initial severity of respiratory failure and subsequent outcomes, has not previously been investigated in acute HRF but has been described in ARDS [10, 21, 45-47]. Notably, a secondary analysis of randomized trial data showed patients with ARDS that rapidly improved within 1 day had substantially lower mortality than patients with persistent ARDS and that $63 \%$ of rapidly improving ARDS patients were moderate or severe at enrollment [10]. A study from the international multicenter LUNG SAFE cohort reported that $82 \%$ of patients with mild ARDS at enrollment have persistent or worsening ARDS in the first week of onset and that patients with persistent/worsening ARDS on ICU day 2 have higher hospital mortality compared to those with resolving ARDS [46]. In general, the $\mathrm{PaO}_{2}: \mathrm{FIO}_{2}$ at onset of respiratory failure is not as consistently associated with overall mortality as $\mathrm{PaO}_{2}: \mathrm{FIO}_{2}$ measured at later time points, and in our study too, we saw the overall mortality among patients with enrollment $\mathrm{PaO}_{2}: \mathrm{FIO}_{2}<150$ was similar to that of 
the entire cohort [48]. These data all support an approach that incorporates reassessing respiratory failure on ICU day 3 to define a subphenotype that may be more pathophysiologically homogenous and at high risk for poor outcomes. Further work is needed to understand which underlying factors contribute to the development of persistent or resolving HRF and their associated differences in mortality, which may relate to treatment (e.g., fluid management, lung-protective ventilation), the patient (e.g., genetic susceptibility to lung injury, chronic comorbidity), and/or the acute illness itself (e.g., pathogen, type of trauma).

Enrollment of patients with persistent HRF into clinical trials of therapies for respiratory failure could help select for patients at higher risk for disease-related outcomes, a strategy known as prognostic enrichment [7]. There is motivation to identify new subsets for prognostic enrichment, since studies of ARDS populations suggest mortality attributable to respiratory failure alone is relatively low $[49,50]$. Similarly, identifying patients with resolving HRF may help avoid exposing them to potentially unnecessary, costly, or toxic new therapies. One strength of our approach is that persistent and resolving HRF can be classified with commonly obtained clinical data, unlike other enrichment strategies requiring measurements only available in research settings $[9,11]$. In addition, we showed that persistent HRF selects for a high-risk population as early as 48 hours after mechanical ventilation, falling into the enrollment window of recent trials and accommodating early therapies for respiratory failure [51].

Another important strategy to improve therapeutic trials is selecting patients with shared biologic mechanisms that are likely to respond to a specific therapy, known as predictive enrichment. Although we do not directly examine whether persistent and resolving HRF have differential responses to therapy, we do show that patients with persistent HRF have higher systemic inflammation and endothelial dysfunction. These patients could be targeted for such emerging therapeutic strategies as early immune modulation and endothelial stabilization/repair [52]. Additionally, persistent HRF enriches for the hyperinflammatory subphenotype of respiratory failure, which among ARDS patients has been associated with differential response to fluid management, statin therapy, and ventilator strategy $[40,41,53]$.

Most notably, we showed that $48 \%$ of persistent HRF patients in the discovery cohort and 54\% in the validation cohort have high mortality and elevated circulating markers of lung injury, yet are not captured in ARDS definitions. Data on these patients with acute HRF who do not meet ARDS criteria remain limited. One large epidemiologic study of acute respiratory failure from Northern
Europe also showed patients who did and did not meet ARDS criteria had similar mortality, although this study was done before the dissemination of lung-protective ventilation and the development of the Berlin definition [5]. Newer work from the LUNG SAFE cohort examined acute HRF patients with new pulmonary infiltrates and also found that patients with unilateral infiltrates (i.e., without ARDS) had similar adjusted mortality to patients with ARDS [27]. In our study, we suspect that the main factor distinguishing \pm ARDS was the chest radiograph, which has been a source of misclassification, measurement burden, and interobserver variability in ARDS diagnosis $[13-16,54,55]$. Our paper builds upon this existing work by also showing that patients with persistent HRF without ARDS had a biomarker profile more consistent with lung injury than ARDS patients with resolving HRF. Non-ARDS patients with persistent HRF may share biologic features with ARDS and thereby may benefit from therapies under investigation for ARDS.

This study has several limitations. First, while we used a broad range of baseline clinical information to control for differences between groups, there may be residual confounding due to unmeasured differences in early clinical care. Second, while the biomarkers measured have been associated with ARDS outcomes and severity, they may not be lung-specific and could reflect processes outside of the lung. Third, the platforms used to measure these biomarkers were not the same in both cohorts. While we would expect this difference to bias our results to the null, it could partially explain discrepancies between cohorts in the relationship between STNFR-1 and persistent HRF. Fourth, while $\mathrm{PaO}_{2}: \mathrm{FIO}_{2}$ is an established measure of the severity of respiratory failure, it is subject to change with ventilator management independent of changes in clinical status $[48,56]$, and captures only a portion of physiologic derangements in $\operatorname{HRF}[56,57]$. Although such differences in ventilator management could have unpredictable effects on classification of persistent HRF, the replication of our main findings in cohorts from two independent medical centers suggests the findings are robust to at least some variability in clinical care. Finally, the identification of persistent HRF is based on a trajectory and cannot be used to classify patients at illness onset. Areas of future research include developing approaches for early discrimination of persistent HRF to aid trial enrollment and designing prospective studies to better understand the mechanisms differentiating persistent from resolving HRF.

\section{Conclusions}

Among mechanically ventilated patients with acute HRF, the subphenotype of persistent HRF on ICU day 3 identifies patients with high mortality and prolonged 
respiratory failure. Patients with persistent HRF have distinctive features early in the course of illness, such as elevated markers of inflammation and endothelial dysfunction. Patients with persistent HRF, both with and without ARDS, may warrant inclusion in trials of targeted therapeutics for respiratory failure.

\section{Supplementary Information}

The online version contains supplementary material available at https://doi. org/10.1186/s13054-021-03755-7.

Additional file 1. Supplemental methods, figures, and tables.

\section{Acknowledgements}

Not applicable.

\section{Authors' contributions}

NAS, CM, CLH, and MMW gave the study concept and design; all authors acquired, analyzed, and interpreted the data, and critically revised the manuscript for important intellectual content; NAS drafted the manuscript; NAS and LRZ carried out the statistical analysis; WCL and MMW supervised the study. All authors read and approved the final manuscript.

\section{Funding}

Authors received funding from the National Heart, Lung, and Blood Institute:T32HL007287, F32HL158088 (NAS); K23HL144916 (EDM); K24HL103836, R01HL135849 (LBW), as well as the National Institute of Diabetes, Digestive and Kidney Disease: K23DK1 16967 (PKB). The funding sources had no role in design and conduct of the study; collection, management, analysis, and interpretation of the data; and preparation, review, or approval of the manuscript.

\section{Availability of data and materials}

The datasets used and/or analyzed during the current study are available from the corresponding authors on reasonable request.

\section{Code availability}

The code used during the current study is available from the corresponding author on reasonable request.

\section{Declarations}

\section{Ethics approval and consent to participate}

The study was approved by the Institutional Review Boards at University of Washington Human Subjects Research Committee and Vanderbilt University Medical Center. Participants were enrolled into the original cohorts by informed consent from patients or their surrogates where possible, or by a waiver of consent, approved by the local institutional review boards for this minimal risk study.

\section{Consent for publication}

Consent for publication was provided by all authors.

\section{Competing interests}

Authors have no competing interests to report.

\section{Author details}

${ }^{1}$ Division of Pulmonary, Critical Care and Sleep Medicine, Department of Medicine, University of Washington, 325 9th Avenue, Box \# 359640, Seattle, WA 98104, USA. ${ }^{2}$ Division of Nephrology, Department of Medicine, University of Washington, Seattle, WA, USA. ${ }^{3}$ Benaroya Research Institute, Seattle, WA, USA. ${ }^{4}$ Sepsis Center of Research Excellence, University of Washington, Seattle, WA, USA. ${ }^{5}$ Division of Allergy, Pulmonary, and Critical Care Medicine, Department of Medicine, Vanderbilt University School of Medicine, Nashville, TN, USA. ${ }^{6}$ Department of Biochemistry and Molecular Medicine, University of Oulu, Oulu, Finland. ${ }^{7}$ Division of Pulmonary and Critical Care, Department of Medicine, Oregon Health and Science University, Portland, OR, USA. ${ }^{8}$ Division of Allergy and Infectious Diseases, Department of Medicine, University of Washington, Seattle, WA, USA. ${ }^{9}$ Department of Pathology, Microbiology and Immunology, Vanderbilt University School of Medicine, Nashville, TN, USA.

Received: 28 June 2021 Accepted: 31 August 2021

Published online: 15 September 2021

\section{References}

1. Rubenfeld GD, Caldwell E, Peabody E, et al. Incidence and outcomes of acute lung injury. N Engl J Med. 2005;353:1685-93. https://doi.org/10. 1056/NEJMoa050333.

2. Herridge MS, Cheung AM, Tansey CM, et al. One-year outcomes in survivors of the acute respiratory distress syndrome. N Engl J Med. 2003;348:683-93. https://doi.org/10.1056/NEJMoa022450.

3. Vincent J-L, Akça S, de Mendonça A, et al. The epidemiology of acute respiratory failure in critically III patients. Chest. 2002;121:1602-9. https:// doi.org/10.1378/chest.121.5.1602.

4. Esteban A, Anzueto A, Frutos F, et al. Characteristics and outcomes in adult patients receiving mechanical ventilation: a 28-day international study. JAMA. 2002;287:345-55. https://doi.org/10.1001/jama.287.3.345.

5. Luhr OR, Antonsen K, Karlsson M, et al. Incidence and mortality after acute respiratory failure and acute respiratory distress syndrome in Sweden, Denmark, and Iceland. Am J Respir Crit Care Med. 1999;159:1849-61. https://doi.org/10.1164/ajrccm.159.6.9808136.

6. Santacruz CA, Pereira AJ, Celis E, Vincent J-L. Which multicenter randomized controlled trials in critical care medicine have shown reduced mortality? A systematic review. Crit Care Med. 2019;47:1680-91. https:// doi.org/10.1097/CCM.0000000000004000.

7. Prescott HC, Calfee CS, Thompson BT, et al. Toward smarter lumping and smarter splitting: rethinking strategies for sepsis and acute respiratory distress syndrome clinical trial design. Am J Respir Crit Care Med. 2016;194:147-55. https://doi.org/10.1164/rccm.201512-2544CP.

8. Semler MW, Bernard GR, Aaron SD, et al. Identifying clinical research priorities in adult pulmonary and critical care: NHLBI working group report. Am J Respir Crit Care Med. 2020. https://doi.org/10.1164/rccm. 201908-1595WS.

9. Calfee CS, Delucchi K, Parsons PE, et al. Subphenotypes in acute respiratory distress syndrome: latent class analysis of data from two randomised controlled trials. Lancet Respir Med. 2014;2:611-20. https://doi.org/10. 1016/S2213-2600(14)70097-9.

10. Schenck EJ, Oromendia C, Torres LK, et al. Rapidly improving ARDS in therapeutic randomized controlled trials. Chest. 2019;155:474-82. https:// doi.org/10.1016/j.chest.2018.09.031.

11. Bos LDJ, Scicluna BP, Ong DSY, et al. Understanding heterogeneity in biologic phenotypes of acute respiratory distress syndrome by leukocyte expression profiles. Am J Respir Crit Care Med. 2019;200:42-50. https:// doi.org/10.1164/rccm.201809-18080C.

12. Bellani G, Laffey JG, Pham T, et al. Epidemiology, patterns of care, and mortality for patients with acute respiratory distress syndrome in intensive care units in 50 countries. JAMA. 2016;315:788-800. https://doi.org/ 10.1001/jama.2016.0291.

13. Rubenfeld GD, Caldwell E, Granton J, et al. Interobserver variability in applying a radiographic definition for ARDS. Chest. 1999;116:1347-53. https://doi.org/10.1378/chest.116.5.1347.

14. Sjoding MW, Hofer TP, Co I, et al. Interobserver reliability of the Berlin ARDS definition and strategies to improve the reliability of ARDS diagnosis. Chest. 2018;153:361-7. https://doi.org/10.1016/j.chest.2017.11.037.

15. Meade MO, Cook RJ, Guyatt GH, et al. Interobserver variation in interpreting chest radiographs for the diagnosis of acute respiratory distress syndrome. Am J Respir Crit Care Med. 2000;161:85-90. https://doi.org/10. 1164/ajrccm.161.1.9809003.

16. Figueroa-Casas JB, Brunner N, Dwivedi AK, Ayyappan AP. Accuracy of the chest radiograph to identify bilateral pulmonary infiltrates consistent with the diagnosis of acute respiratory distress syndrome using computed tomography as reference standard. J Crit Care. 2013;28:352-7. https://doi.org/10.1016/j.jcrc.2012.12.002.

17. Lichtenstein D, Goldstein I, Mourgeon E, et al. Comparative diagnostic performances of auscultation, chest radiography, and lung 
ultrasonography in acute respiratory distress syndrome. Anesthesiology. 2004;100:9-15. https://doi.org/10.1097/00000542-20040 1000-00006.

18. Pham T, Rubenfeld GD. Fifty years of research in ARDS. The epidemiology of acute respiratory distress syndrome. A 50th birthday review. Am J Respir Crit Care Med. 2017;195:860-70. https://doi.org/10.1164/rccm. 201609-1773CP.

19. Béduneau G, Pham T, Schortgen F, et al. Epidemiology of weaning outcome according to a new definition. The WIND study. Am J Respir Crit Care Med. 2016;195:772-83. https://doi.org/10.1164/rccm. 201602-03200C

20. Burnham EL, Janssen WJ, Riches DWH, et al. The fibroproliferative response in acute respiratory distress syndrome: mechanisms and clinical significance. Eur Respir J. 2014;43:276-85. https://doi.org/10.1183/ 09031936.00196412.

21. Sanchez E, Price DR, Chung K-P, et al. Persistent severe acute respiratory distress syndrome for the prognostic enrichment of trials. PLOS ONE. 2020;15:e0227346. https://doi.org/10.1371/journal.pone.0227346.

22. Peyrani P, Arnold FW, Bordon J, et al. Incidence and mortality of adults hospitalized with community-acquired pneumonia according to clinical course. Chest. 2020;157:34-41. https://doi.org/10.1016/j.chest.2019. 09.022.

23. Bhatraju PK, Mukherjee P, Robinson-Cohen C, et al. Acute kidney injury subphenotypes based on creatinine trajectory identifies patients at increased risk of death. Crit Care. 2016;20:372. https://doi.org/10.1186/ s13054-016-1546-4.

24. Ross JC, Castaldi PJ, Cho MH, et al. Longitudinal modeling of lung function trajectories in smokers with and without chronic obstructive pulmonary disease. Am J Respir Crit Care Med. 2018;198:1033-42. https://doi.org/10.1164/rccm.201707-14050C.

25. Su C, Xu Z, Hoffman K, et al. Identifying organ dysfunction trajectorybased subphenotypes in critically ill patients with COVID-19. Sci Rep. 2021;11:15872. https://doi.org/10.1038/s41598-021-95431-7

26. Bhavani SV, Carey KA, Gilbert ER, et al. Identifying novel sepsis subphenotypes using temperature trajectories. Am J Respir Crit Care Med. 2019;200:327-35. https://doi.org/10.1164/rccm.201806-11970C.

27. Pham T, Pesenti A, Bellani G, et al. Outcome of acute hypoxaemic respiratory failure: insights from the LUNG SAFE study. Eur Respir J. 2021;57:2003317. https://doi.org/10.1183/13993003.03317-2020.

28. Mikacenic C, Price BL, Harju-Baker S, et al. A two-biomarker mode predicts mortality in the critically III with sepsis. Am J Respir Crit Care Med. 2017:196:1004-11. https://doi.org/10.1164/rccm.201611-2307OC

29. Bhatraju PK, Robinson-Cohen C, Mikacenic C, et al. Circulating levels of soluble Fas (sCD95) are associated with risk for development of a nonresolving acute kidney injury subphenotype. Crit Care. 2017. https:// doi.org/10.1186/s13054-017-1807-x.

30. Singer M, Deutschman CS, Seymour CW, et al. The third international consensus definitions for sepsis and septic shock (sepsis-3). JAMA. 2016:315:801-10. https://doi.org/10.1001/jama.2016.0287.

31. Ware $L B$, Koyama T, Zhao Z, et al. Biomarkers of lung epithelial injury and inflammation distinguish severe sepsis patients with acute respiratory distress syndrome. Crit Care. 2013;17:R253. https://doi.org/10. $1186 / \mathrm{cc} 13080$

32. Rice TW, Wheeler AP, Bernard GR, et al. Comparison of the Spo2/Fio2 ratio and the Pao2/Fio2 ratio in patients with acute lung injury or ARDS. Chest. 2007;132:410-7. https://doi.org/10.1378/chest.07-0617.

33. National Heart, Lung, and Blood Institute PETAL Clinical Trials Network, Moss M, Huang DT, et al. Early neuromuscular blockade in the acute respiratory distress syndrome. N Engl J Med. 2019;380:1997-2008. https://doi.org/10.1056/NEJMoa1901686.

34. McAuley DF, Laffey JG, O'Kane CM, et al. Simvastatin in the acute respiratory distress syndrome. N Engl J Med. 2014;371:1695-703. https:// doi.org/10.1056/NEJMoa1403285.

35. Fowler AA, Truwit JD, Hite RD, et al. Effect of vitamin C infusion on organ failure and biomarkers of inflammation and vascular injury in patients with sepsis and severe acute respiratory failure: the CITRIS-ALI randomized clinical trial. JAMA. 2019;322:1261-70. https://doi.org/10. 1001/jama.2019.11825.

36. Acute respiratory distress syndrome: the Berlin definition / Critical Care Medicine | JAMA | JAMA Network. https://jamanetwork.com/journals/ jama/fullarticle/1160659. Accessed 16 Mar 2020.
37. Zou G. A modified Poisson regression approach to prospective studies with binary data. Am J Epidemiol. 2004;159:702-6. https://doi.org/10. 1093/aje/kwh090.

38. Knaus WA, Wagner DP, Draper EA, et al. The APACHE III prognostic system. Risk prediction of hospital mortality for critically ill hospitalized adults. Chest. 1991;100:1619-36. https://doi.org/10.1378/chest.100.6. 1619

39. Knaus WA, Draper EA, Wagner DP, Zimmerman JE. APACHE II: a severity of disease classification system. Crit Care Med. 1985;13:818-29.

40. Sinha P, Delucchi KL, Thompson BT, et al. Latent class analysis of ARDS subphenotypes: a secondary analysis of the statins for acutely injured lungs from sepsis (SAILS) study. Intensive Care Med. 2018;44:1859-69. https://doi.org/10.1007/s00134-018-5378-3.

41. Famous KR, Delucchi K, Ware LB, et al. Acute respiratory distress syndrome subphenotypes respond differently to randomized fluid management strategy. Am J Respir Crit Care Med. 2017;195:331-8. https://doi.org/10.1164/rccm.201603-06450C.

42. Kitsios GD, Yang L, Manatakis DV, et al. Host-response subphenotypes offer prognostic enrichment in patients with or at risk for acute respiratory distress syndrome*. Crit Care Med. 2019;47:1724-34. https://doi. org/10.1097/CCM.0000000000004018.

43. Heijnen NFL, Hagens LA, Smit MR, et al. Biological subphenotypes of ARDS show prognostic enrichment in mechanically ventilated patients without ARDS. Am J Respir Crit Care Med. 2021. https://doi.org/10. 1164/rccm.202006-25220C.

44. Sinha P, Churpek MM, Calfee CS. Machine learning classifier models can identify ARDS phenotypes using readily available clinical data. Am J Respir Crit Care Med. 2020. https://doi.org/10.1164/rccm. 202002-03470C.

45. Madotto F, Pham T, Bellani G, et al. Resolved versus confirmed ARDS after $24 \mathrm{~h}$ : insights from the LUNG SAFE study. Intensive Care Med. 2018;44:564-77. https://doi.org/10.1007/s00134-018-5152-6.

46. Pham T, Serpa Neto A, Pelosi P, et al. Outcomes of patients presenting with mild acute respiratory distress syndrome: insights from the LUNG SAFE study. Anesthesiology. 2019;130:263-83. https://doi.org/10.1097/ ALN.0000000000002508.

47. Villar J, Fernández RL, Ambrós A, et al. A clinical classification of the acute respiratory distress syndrome for predicting outcome and guiding medical therapy*. Crit Care Med. 2015;43:346-53. https://doi.org/ 10.1097/CCM.0000000000000703.

48. Villar J, Pérez-Méndez L, Blanco J, et al. A universal definition of ARDS: the $\mathrm{PaO}_{2} / \mathrm{FiO}_{2}$ ratio under a standard ventilatory setting - a prospective, multicenter validation study. Intensive Care Med. 2013;39:583-92. https://doi.org/10.1007/s00134-012-2803-x.

49. Fuchs $L$, Feng $M$, Novack $V$, et al. The effect of ARDS on survival: do patients die from ARDS or with ARDS? I Intensive Care Med. 2019;34:374-82. https://doi.org/10.1177/0885066617717659.

50. Auriemma CL, Zhuo H, Delucchi K, et al. Acute respiratory distress syndrome-attributable mortality in critically ill patients with sepsis. Intensive Care Med. 2020;46:1222-31. https://doi.org/10.1007/ s00134-020-06010-9.

51. Huang DT, Angus DC, Moss M, et al. Design and rationale of the reevaluation of systemic early neuromuscular blockade trial for acute respiratory distress syndrome. Ann Am Thorac Soc. 2017;14:124-33. https://doi.org/10.1513/AnnalsATS.201608-6290T.

52. Horie S, McNicholas B, Rezoagli E, et al. Emerging pharmacological therapies for ARDS: COVID-19 and beyond. Intensive Care Med. 2020;46:2265-83. https://doi.org/10.1007/s00134-020-06141-z.

53. Calfee CS, Delucchi KL, Sinha P, et al. Acute respiratory distress syndrome subphenotypes and differential response to simvastatin: secondary analysis of a randomised controlled trial. Lancet Respir Med. 2018;6:691-8. https://doi.org/10.1016/S2213-2600(18)30177-2.

54. Pham T, Pesenti A, Bellani G, et al. Outcome of acute hypoxaemic respiratory failure. Insights from the lung safe study. Eur Respir J. 2020. https://doi.org/10.1183/13993003.03317-2020.

55. Kotok D, Yang L, Evankovich JW, et al. The evolution of radiographic edema in ARDS and its association with clinical outcomes: a prospective cohort study in adult patients. J Crit Care. 2020;56:222-8. https:// doi.org/10.1016/j.jcrc.2020.01.012.

56. Villar J, Pérez-Méndez L, López J, et al. An early PEEP/FiO 2 trial identifies different degrees of lung injury in patients with acute respiratory 
distress syndrome. Am J Respir Crit Care Med. 2007;176:795-804. https://doi.org/10.1164/rccm.200610-15340C.

57. Morales-Quinteros L, Schultz MJ, Bringué J, et al. Estimated dead space fraction and the ventilatory ratio are associated with mortality in early ARDS. Ann Intensive Care. 2019;9:128. https://doi.org/10.1186/ s13613-019-0601-0

\section{Publisher's Note}

Springer Nature remains neutral with regard to jurisdictional claims in published maps and institutional affiliations.
Ready to submit your research? Choose BMC and benefit from:

- fast, convenient online submission

- thorough peer review by experienced researchers in your field

- rapid publication on acceptance

- support for research data, including large and complex data types

- gold Open Access which fosters wider collaboration and increased citations

- maximum visibility for your research: over 100M website views per year

At BMC, research is always in progress.

Learn more biomedcentral.com/submissions 